\title{
Screen navigation system for visually impaired people
}

\author{
James Francis Ohene-Djan and Sandra A. Fernando
}

\begin{abstract}
Purpose - The SETUP09 system consists of both navigation and a computer-aided drawing technique for the people who are blind and visually impaired (BVI). The purpose of this paper is to address the need for a screen navigation technique, which can facilitate a user's ability to produce art, and scientific diagrams electronically, by introducing a compass-based screen navigation method.

Design/methodology/approach - BVI computer users were tested using different screen navigation tasks to assess the accuracy and efficiency of this compass-based navigation technique by using a prototype (SETUP09) and tactile paper grid maps.

Findings - The results confirmed that the compass-based navigation facilitates higher accuracy in screen-based moving and location recognition with a noticeable reduction in time and effort.

Research limitations/implications - Additional improvements such as the addition of a sound layer to the interface, use of hotkeys, braille and user speech inputs are yet to be tested.

Social implications - The current lack of suitable and efficient screen navigation technology is a limiting factorforBVIstudents and computer users in producing diagrams and drawings. This may place limitations on their careerprogression and life contentment. It is challenging for a BVI person to draw diagrams and art, which are commonly taught in education or used in industry. The compass-based screen navigation system was developed to address BVI users' need to be able to create such content.

Originality/value - A compass-based navigation method enables screen navigation through a formal command language and enables intuitive movement to a screen location using matrix-style compass directions with zoom-in and zoom-out capabilities.

Keywords Assistive technology, Bind users, Blind drawing, Compass-based navigation,

Screen navigation technology, Visually impaired

Paper type Research paper
\end{abstract}

\section{Introduction}

The lack of efficient drawing and screen navigation in assistive technologies is a major hindrance for individuals who are blind and visually impaired (BVI) in education, work and life in general (Hershetal., 2008). For example, adrawing should enabletheuserto memoriseafloor plan, easily navigate, relocate and backtrack to the original point, and memorise object arrangement, minimising the processing of information and the presence of repetition of content in different formats, be easy to learn and easy to use, and take less time to complete a task (Hersh et al., 2008). Most BVI students and practitioners are in the habit of using tactile maps to recognise highlight-raised line art or objects (Takagi, 2009). However there are limitations to the information that tactile graphics can convey. Since Bach-Y-Rita presented the idea of tactile-vision sensory substitution in 1969, similar technology applications have seen rapid growth. From tactile-vision perception and understanding, to voice-vision substitution, this has been incorporated in various ways, helping BVI people in their daily living, academic lives and careers (Rita, 2004). Even though tactile images and 3D printing exists, this technology needs further improvement with complex and dynamic art production (Williams et al., 2014).

Differentscreennavigationmethodshave been experimentedinthepast(Kameland Landay, 2002; ZhuandFeng,2010; Searsetal.,2002; FengandSears, 2004).Kamal(KamelandLanday,2002) 
hasintroducedagrid-basednavigationtechniquethat dividesthe screenintoninenavigablesmaller workspaces. Zhu et al. (Zhu and Feng, 2010) have also introduced enhancement to gridbased navigation by proposing magnification and fine tuning. A continuous direction-based solution was proposed by Sears (Sears et al., 2002) in 2002 where the user initiates the movement of the cursor and the cursor moves to the initiated direction until it is controlled by another command. A target-based cursor (Feng and Sears, 2004) takes advantage of labels on the document and moves the cursor to the label initiated by the user. This technique mostly works on text documents or labelled applications.

We proposed a compass-based navigation method that enables the screen navigation through formal language commands (Ohene-Djan and Fernando, 2016). A compass on the interface takes the user to the intended location, where the screen is divided in to $3 \times 3$ memorable locations such as north, south, east, west, north-west, north-east south-east and southwest. The granularity is changed by zoom in and zoom out commands. When zoom in is executed, the current location is subdivided into another $3 \times 3$ smaller locations. Inputs invoke the primitive objects, locations and operations. Audio feedback is given for confirmation and verification. A set of keywords is used to form the language for navigation.

In this paper we present the findings of a study to access the accuracy and efficiency of compass-based screen navigation using experimentation conducted on a group of BVI subjects. Efficiency was measured by task completion time, ease of use was measured by the number of errors made, and accuracy was measured relative to tactile maps.

This paper is structured as follows: a general overview of related research of screen navigation techniques; the method and procedure of our experiments; analysis of the results of error rate and completion time; and concludes with feedback and broader implications.

\section{Related research}

Screen navigation could be a time consuming activity for blind and partially sighted individuals. Multimodal techniques are proven to be more effective when using a keyboard, stylus or speech (Suhm et al., 2001), but this is not always viable. Hands-free speech-based solutions can allow faster and more robust experiences that traditional keyboards cannot (Sears et al., 2001).

Several navigation mechanisms were previously introduced to navigate text documents. Speech-based easy access to graphical interfaces was introduced by de Mauro (de Mauro et al., 2001) using a voice-controlled mouse. Subsequently direction-based navigation techniques manifest discrete or continuous cursor movements. For example, in discrete direction-based cursor movements, a "move left two words" command causes the cursor to find the new location by jumping two words left. Discrete direction-based commands can also be set as inches, centimetres or letters on the screen. Continuous direction-based navigation primarily trigged by speech inputs ensures start and stop commands. For example, "move right" moves the cursor to the right until the "stop" command is given. Since the cursor can move horizontally or vertically, such continuous cursor movements to a diagonal location is associated with higher error rate. Screen distance traversed has impacted on selection time and errors. There is an additional complexity: with increased distance, the cursor travelled before the "stop" command was executed (Sears et al., 2001).

Mcnair and Waibel (1994) have investigated and introduced an early version of target-based speech navigation, as well as immersed speech-based navigation on web documents. A user says a word that serves as a link to other web pages or menus or by saying a number that eventually takes them to another link. With target-based navigation, a speech command "Select Friday" in the text document can directly navigate to the word Friday. It also can be set to navigate to icons, menus and regions on the screen. Even though this resulted in

a minimum number of errors, it has a longer task completion time. There is also a difficulty with the higher number of similar possible targets and the user needing to know required targets in the document.

Agrid-basednavigationmethod was first studied byKamal(Kamel and Landay, 2002)and finetuning and magnification research was studied by Feng and Zhu (Zhu and Feng, 2010). The gridbased navigation system manoeuvres to an area on the screen without contextual information, 
using $3 \times 3$ matrix system navigation techniques. The formation of a nine-cell system works well with the intuition of BVI individuals as it is organised similar to a telephonekeypad. The cell numberswork from left to right and top to bottom. The system enables users to move to and from any of the nine cells. The uniquepoint of a cell is the centrepoint, whichis then selectedto perform drawing. Different pallet selection tools such as shape, type, colour and commands for saving support the main functionalities of drawing. Each pallet provides nine choices where a user is able to navigate by selecting the pallet options. A grid recursive schema allows users to make more precise selections. Users can find a unique position by further dividing into nine more cells and increasing zoom levels. Users can label the object for identification.

We introduce a compass-based navigation system, which is similar to Kamal's matrix system. It is recorded that the user spends a considerable portion of time navigating the screen from one location to another within the document they create when using a navigation system such as hands-free or speech-based (Sears et al., 2002). Therefore the alternative screen navigation technique is introduced in this paper and tested for efficiency. The cells are not identified by numbers but compass directions such as north, south, east, west, northwest, north-east, south-east and south-west. Compass-based navigation also has nine unique points in a cell rather than one centre unique point as in the grid-based navigation system. The system is named "SETUP09". The system has drawing capability by user direct command input unlike the pallet options in the grid-based navigation system for example: "zoomin centre", or "zoomin north". Drawing commands are either pre-defined or user defined, and they also can be used later by simply calling its label. Speech input mechanism is one of many interfaces for input selections such as a keyboard, speech and braille. Multimodal verification and output mechanisms are still in experimentation.

"SETUP09" is a computer aided navigation and drawing system for BVI people. Drawing technologies and image analysis systems were introduced in the past, without much focus on layout and navigation. Navigation systems such as a direction-based navigation system, a target-based navigation system, a grid-based navigation system or drawing systems such as KEVIN (Blenkhorn and Evans, 1998), PLUMB (Calder et al., 2007), or image analysis systems such as TeDUB (Petrie et al., 2002), do not explore their ability to build a cognitive map of the navigation. Therefore, a systematic approach is required to design and develop the understanding of a floor plan of such navigation technology for BVI people. This paper proposes experimenting with a systematic navigation technique for BVI users using compass- based cursor control systems to support efficient navigation.

\section{Methodologies}

Experiments were conducted on a group of BVI people to find out the efficacy of compass-based navigation solutions used by individuals who are totally or severely blind. A group of five BVI different age groups, different levels of blindness and computer literacy are utilised. A compass-based navigation solution, SETUP09 system is proposed to navigate in a virtual environment. The computer uses a speech-based feedback mechanism. Given the low number of participants available, it is understood that the most productive method to collect data is through one to one training, experiment observation and recording. The age of the participants ranges from 27 to 52 . Computer literacy ranges from moderate to high. Computer literacy was measured by participants' confidence using computers at work, confidence using a keyboard, and confidence using word processing applications. Three participants are totally blind and two severely blind where sight is barely limited to lines and shapes.

Experiment 1 (task 1-5)testifies the alternative hypothesis: going through a virtual environment generates a navigation map in a BVI person's mind. Experiment 2 (task 6) testifies the alternative hypothesis: SETUP09 navigation method is an efficient method to find a specific location on the screen compared to tactile maps. Efficiency is measured by task completion time; ease of use is measured by error rates and tactile maps (pictured in Figure 1) are used to measure accuracy.

An investigation has been carried out to find out whether going through a virtual environment generates a cognitive map in a BVI person's mind. Cognition is measured by reassuring the understanding of the target location, for example tasks to find a given location on the SETUP09 


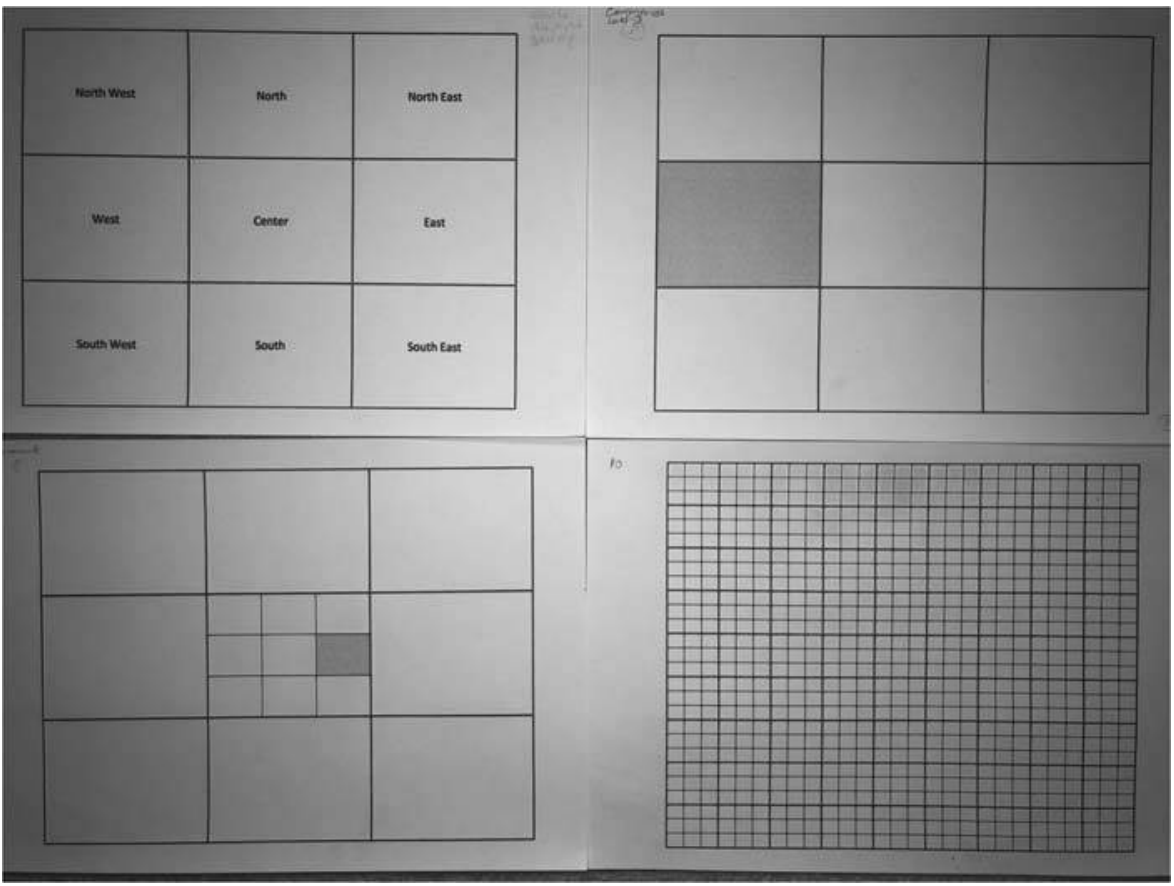

system, or tasks to recognise a given target location on a tactile grid map. Location accuracy is measured by error rate. A lower error rate corresponds with increased accuracy. Age and computer literacy may impact performance. Therefore, the following group assumptions are examined: some participants might spend more time completing the task; some participants might make more errors than others; and some participants might find it difficult to demonstrate their cognition on the tactile map/swell paper. Swell paper is a special kind of paper upon which images and art can be printed or sketched and turned in to tactile images or letters. It can be used with a marker pen, printer or photocopier to create images. When the paper is subjected to a very bright light such as a Zyfuse heating machine the dark areas create raised relief lines.

To select a target location, a user must enter/input zoomin command in the area intended to travel. Thezoomlevelinfluencesthesize of the targetas itdivides thescreenintonine sections. Each time the zoom is used, the zoom level increases by one. This study only discusses the navigation efficacy of compass-based navigation, whereas in future studies the efficacy of the drawing commands will be investigated. There are five target locations in experiment 1. Each time, the screen starts with the default start position, with nine memorable locations (e.g. north, south, east, west, northeast, north-west, south-east or south-west). Object size and the screen location are determined by the "zoomin" command. It takes the focus into smaller granularity on the screen. $\mathrm{N}, \mathrm{Nand}$ Cinform the area of the screen, which are north, north and centre as captured in Figure 2 .

\section{Implementation}

A MacBook Pro (Retina, 13-inch) with Mac and Windows operating system, Intel Iris Graphics $61001536 \mathrm{MB}$ and FreeTTS (speech synthesis system) is used for speech output interaction. SETUP09 is a language built on a Java compiler, which recognises and processes drawing commands typed by the user. Before completing the task each participant was given a user manual and a training session. During the training period, participants used the available commands to test different screen target locations of their choice. No time limit was set for the training session. They could repeat it if desired. Assistance was available during training. Needless to say, they rarely asked for help. After the training six different target locations were designed to navigate. Each participant 


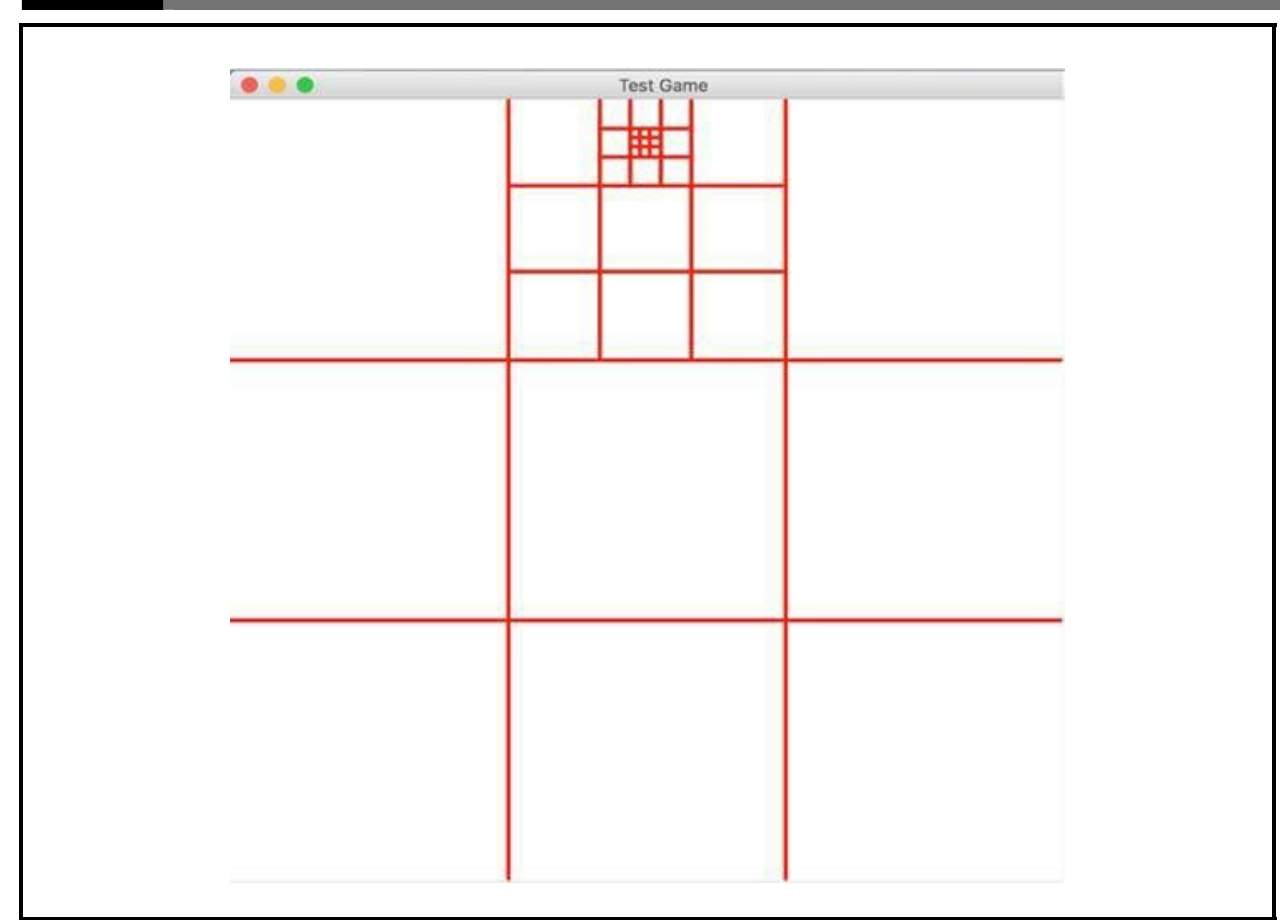

spent around 70 minfor the training and for the experiment and they were given incentives: each participant was given five pounds for expenses. After the experiment, they completed the questionnaire regarding their satisfaction of the experiment.

Participant on Plate 1 is recognising the highlighted areas on the swell/tactile paper, and the participant on Plate 2 is inserting commands into the SETUP09 system.

The first study investigated that going through a virtual environment generates a navigation map in a blind person's mind. As mentioned in therelated research, screennavigation doesnotalwayslead to accurate cursor location where BVI users intend to travel. The user must know and remember thecommands, in ordertotype orspeak therequiredcommands. The system must thenrecognise words typed or spoken. When using compass-based navigation this delay can cause errors or incurincorrectcognitivemaps of thescreennavigationinBVlusers. Asa solution, thesystem could provide some assistance by informing the direction and the zoom level. More specifically, this study investigated the potential benefits of compass-based navigation as well as investigating the accuracy of compass-based navigation comparing with a tactile map, and efficiency using completion time. For this study, we implement thenavigation system as follows:

1. Variables:

- independent variable is the blind user group; and

- thedependentvariableincludes completion time, error rate, tactilemapaccuracyanduser satisfaction.

2. Completion time is the start time to the end time to navigate to the desired location.

3. Accuracy is calculated on the number of correct and incorrect attempts.

4. Efficiency is calculated by the time taken to complete the same activity using the prototype SETUP09 system, tactile papers and standard papers.

5. Cognition is demonstrated by cross-checking the understanding/cognition of system target locations using a tactile grid map or vice versa. A short questionnaire using a fivepoint scale (one as the most positive and five as the most negative) was used to access the participant's perception of accuracy, ease of use and cognition after the experiment. 


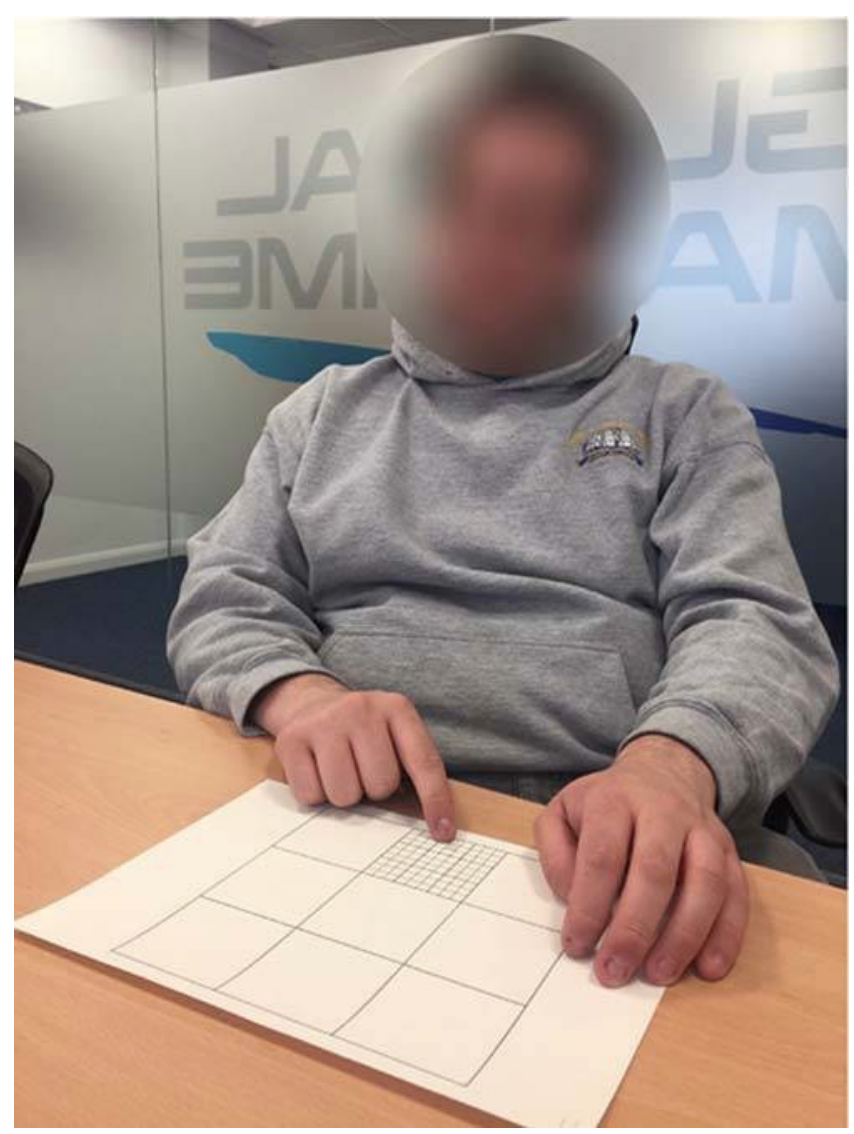

Plate 2 Participant using SETUP09 system

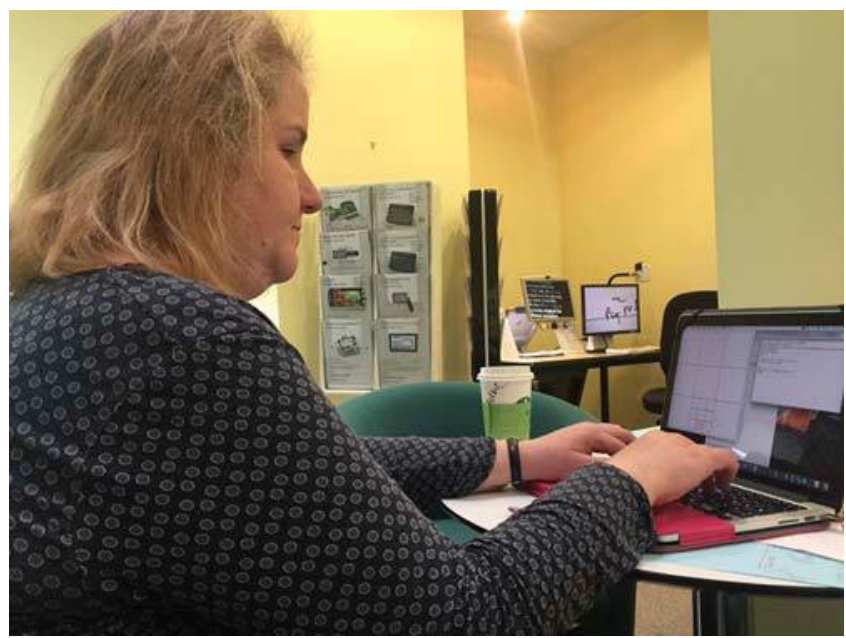


A short usability questionnaire was designed based on two basiccriteria detailed in the research hypothesis, finding the effectiveness (ability to find a target location) and efficiency (ability to build a cognitive map) of compass-based navigation system:

1. Zoominolocation $\mathrm{W}$ : locations can be north, south, east, west, north-east, north-west, south-east, south-west or centre. The command is used to navigate to a specific and smaller screen location.

2. Zoomout: this command is used extract from a specific area of the screen.

3. My position: this is a helper command to trace the location of the screen in its current position.

At the start, the cursor orthe point of focus sets at the top leftcornerposition and when a new command is executed the cursor or the point of focus moves. To draw a line or write a label, the user can call a specific point on the focus area or system by default. This then sets its new cursor position to the left most position of the area that populates by zoomin command based on the level of zoom. In case of incorrect entry, a user can abort input commands. Figure 3 demonstrates the focus area of the command Zoomin N:

\section{- Zoomin oNW}

\subsection{Navigation on the screen}

For example, if the intended navigation location is the south centre of the east location, the navigation path is East $\rightarrow$ Centre $\rightarrow$ South (Figure 4).

\subsection{Procedure}

4.2.1 Experiment 1. Experiment 1 sought to identifythe accuracy and the efficiency of the screen navigation technique using tactile paper grid maps and compass-based navigation systems (SETUP09). Participants used swell papers to recognise a specific location. Swell paper is a capsule paper or a puff paper, which is used for the production of tactilegraphics orimages for BVI people. Theywere asked to identifyan arearaised, construct a navigation map, identifyand input navigation commands and input those commands to bring the cursor to a specificlocation using SETUP09. Participants were also asked to find a given screen location on the system with and without the use of swell papers. Participants were asked to type the commands to go back to the starting point. The purpose of the activity was to find out if participants were able to navigate to a specific location on the computer screen that is given to them and trace their way back.

\section{Figure 3 System SETUP09: focus area on the north}

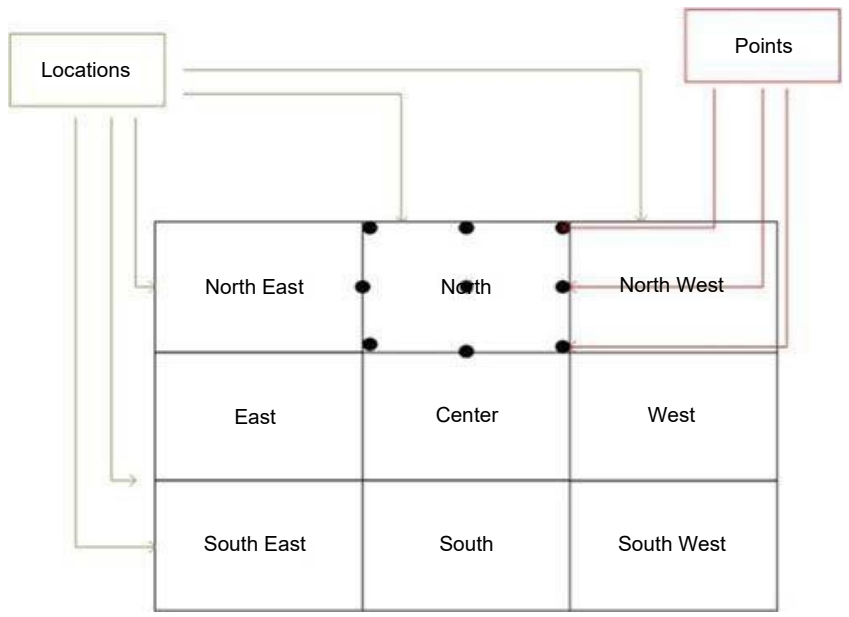




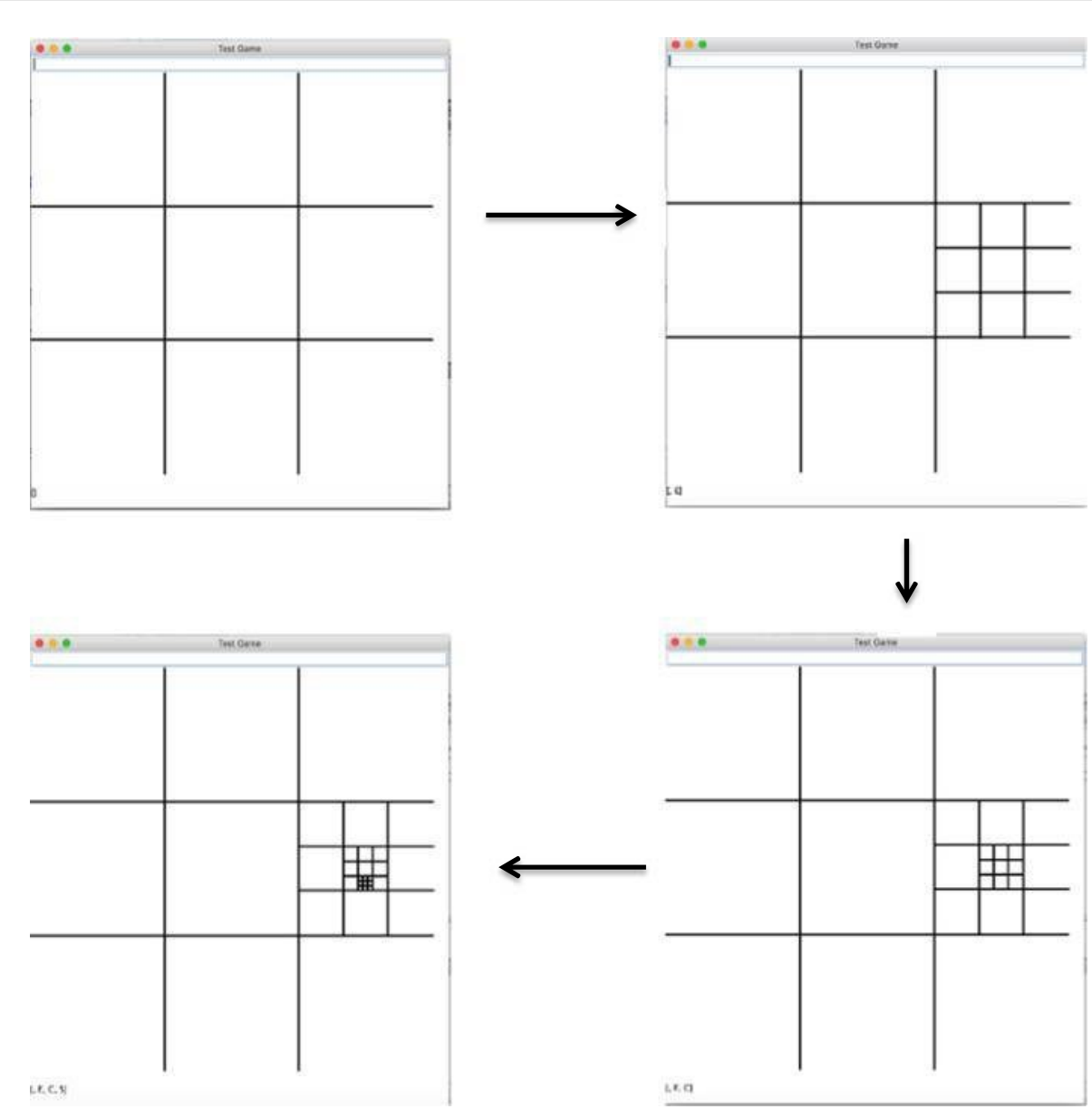

Notes: Step 1 - initial screen; step 2 - initial screen to east location; step 3 - east to east-centre; step 4 - east-centre to east-centre-south

Some locations were smaller than others, with activities designed to target different zoom levels (2, 3). Each participant had five trails of experiment 01 ; accuracy and timewere recorded.

4.2.2 Experiment 2. For experiment2, participants were asked to find the centre bydrawing a circle shape on a given resource. For thisexperiment, we used A4 paper, swell paperwith agrid system and the SETUP09 system. Participants were asked to draw a circle on the centre of the given resource and the time and accuracy were recorded. The purpose of the activity was to assess the efficiency of different techniques to find a specific location on the computer screen. An easy targetwas givenforthetasktolimitpossibleconfusions. Eachparticipanthadatrail of experiment2.

4.2.3 Evaluation. Participants' feedback on satisfaction and usability was collected and recorded from both experiments.

4.2.4 Results. The results are based on completion time of the experiment, errors made and accuracy as dependent variables, and the user as the independent variable.

\subsection{Completion time}

4.3.1 Time taken foractivity 1-5. All participants were able to complete the first five activities with the average time of 33 (ss). Some participants were quick to learn and other participants took time to find the location on the swell paper. All of them found grids on swell papers were tougher 
than the SETUP09 system as they had to navigate through remembering and counting grids. Theyexpended more time during activity 4 and activity 5 , speciallywhere the zoom level extends beyond two levels. Severelyblind participants were able to identifytheraisedline printing quicker than totally blind participants. They all enjoyed the activities and were keen to get it right. Blind participants spent no longer than 1.13 (m:ss) with the standard deviation $S^{1} / 40.22$ (ss).

4.3.2 Time taken for activities on level 2 and 3. Participants were able to reach target locations on the SETUP09 system much faster than when using tactile maps. Participants spent an average time of 0.34 (m:ss) on the SETUP09 system as opposed to spending 0.42 (m:ss) to find the location using the tactile paper reaching zoom levels 2 and 3 activities. It is evidenced that a system-generated cognitive map enabled them to find a location much faster than a tactile map. One participant made a mistake typing the command, hence took a longer time completing an activity on the SETUP09 system, and another participant made a mistake looking for a location on the tactile paper incorrectly orientated, hence took more time using the tactile paper. Overall, participants found it easier to build mental navigation models faster with the SETUP09 system than grids on tactile papers.

Challenges. Interpreting tactile images was difficult for participants where there were more than two zoom levels presented. Some participants had to attempt this several times due to the need to count smaller areas on swell papers to recognise the exact map location or map the given commands on swell papers. However, they all appeared to be comfortable with the SETUP09 locationfinder.Everyparticipantmentioned that thenavigationmap canbevisualisedaccurately; however, relating it onto the grids on swell paper was more difficult.

\subsection{Accuracy}

4.4.1 Accuracy of the cognitive map. Table I shows that 72 per cent of attempts were successful, and participants showed the exact same location on a tactile swell paper going through the same compass-based navigation technique, while 28 per cent of attempts were unsuccessful at the first effort. However, they managed to self-correct by probing the system, and using commands such as "My position" to complete the activity.

Seven errors were made out of 25 input commands. Each participant had five input commands and there were five participants. On average they made at least one error and at most two errors. Some of them were confused with raised line grids as some activities were designed to identity the target location highlighted on swell papers. It is mainly due to closely printed grids on the tactile grids and it seemed difficult to identify exact locations on anything beyond second zoom level. However, they all were confident about their mental models and trusted the SETUP09 system to navigate to the correct destination. The errors were predominantly made during activities 4 and 5 when recognising the exact location highlighted on swell papers that was located on the third zoom level.

4.4.2 Incorrect understanding of activity 1-5 (wrong cognition). Total errors made are visualised using a bar chart, Figure 5 . Seven errors were made and they were mostly on zoom level 3 related activities. The main reason for the zoom level 3 errors was due to the fact that participants had to countandrememberthegrids onthetactilepaper. Thisimposedhighcognitiveloadand confusion.

\section{Table I Number of correct and incorrect recognitions made during activity 1-5}

Participants

Participant 1

Participant 2

Participant 3

Participant 4

Participant 5
Number of correct recognitions

Numberofincorrect recognitions 


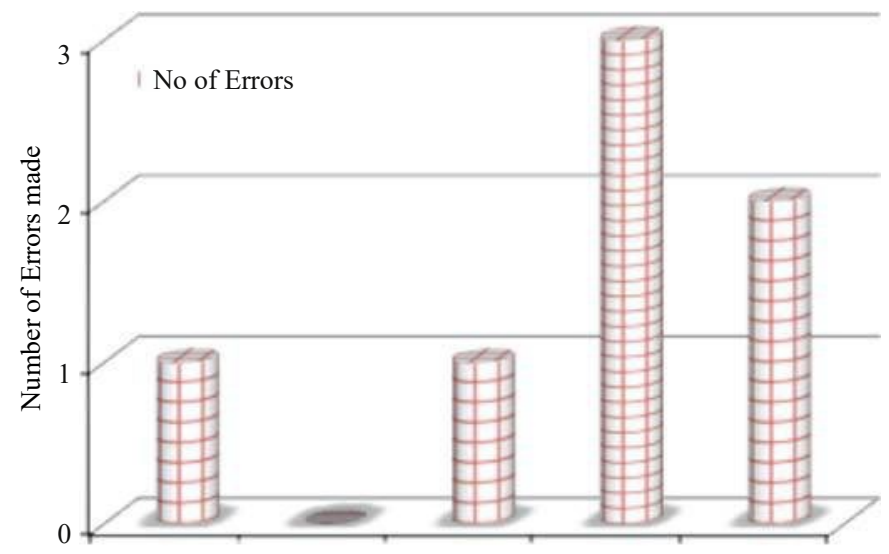

Activity 1 Activity 2 Activity 3 Activity 4 Activity 5 Activities

4.4.3 Accuracy of the cognitive map of different zoom levels. Figure 6 illustrates the nature of errors made during task 1 to 5 . Participants made two errors on zoom level 2 activities and five errors on level 3 activities. More errors were made on activities related to zoom level 3 compared to zoom level 2.

The longer time was taken to complete the task where errors were presented when completing certain activities. More errors were made on activities 4 and 5 , which had three zoom levels to recognise. Errors are labelled E1-E7.

\subsection{Efficiency}

4.5.1 Time taken for activity 6 . Finding the centre area of a swell paper, A4 paper and SETUP09 system.

Finding the centre region on swell papers andSETUP09was easy. But finding thecentre on A4 paper was not accurate in any of their attempts. All of them demonstrated a nearby area but not the exact centre on A4 and participants knew they were wrong (Figure 7).

\section{Fiğ ure 6 Time and errors during activity $1-5$}

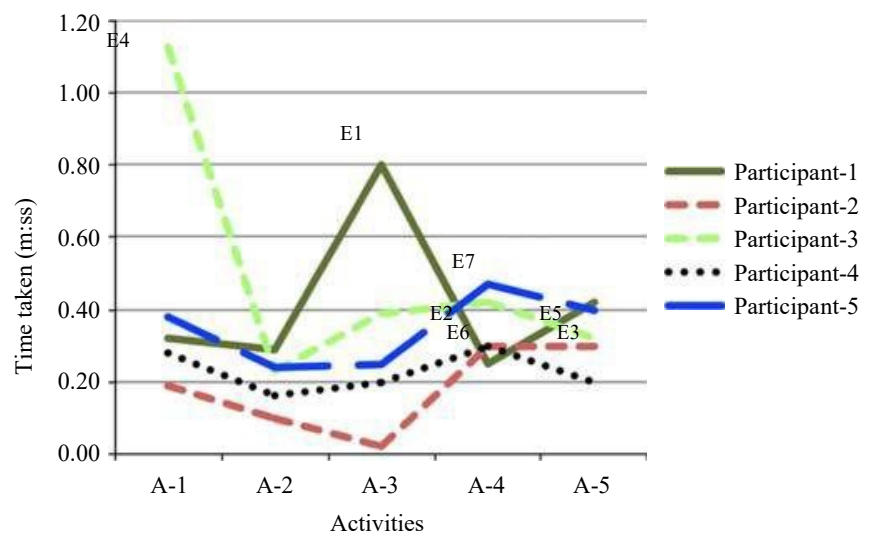




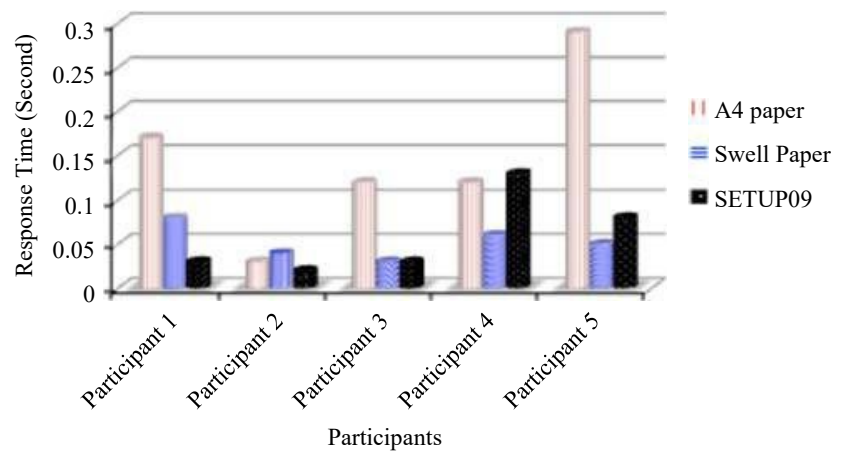

All participants were able to recognise the centre area on the swell paper prettywell compared to theSETUP09 system. Itcould be that the swell paperwas onlydesigned tothe firstzoomlevel with nine areas. Chart 12 suggests that all participants managed to find the centre area on the SETUP09 system faster compared with the paper method and they were highly confident of their accuracy. On average, finding the centre on A4 paper took them 14 (ss), finding the centre area on swell paper took them 5 (ss) and finding the centre on the system took them 5 (ss).

4.5.2 Ranking activity 6. Finding the centre area of a swell paper, A4 paper and SETUP09 system, 1 being the easiest and 10 being the most difficult. Participants found it easy to use the SETUP09 system and swell paper to find location C (Centre). But identifying location C using the A4 paper proved to be difficult. Participants were highlysatisfied and confident of the accuracy of the swell paper and SETUP09. This is illustrated using a line graph, Figure 8.

\section{Findings}

This observation demonstrates the successful building of navigation maps of BVI participants through a compass-based navigation system without the help of a support worker. For the purpose of this experiment the facilitator initiated the SETUP09 application for the participants, answered the participants' questions about the software interface, training demonstration and exercises. Many of the participants were interested in the nature of the navigation movement, for example, whether specific commands would move the cursor to the target location as desired. Participants were also able to construct navigation models when several zoomin commands take place within the same command line execution. Another observation was that some participants

\section{Figure 8 User satisfaction-1 being the easiest and satisfied method}

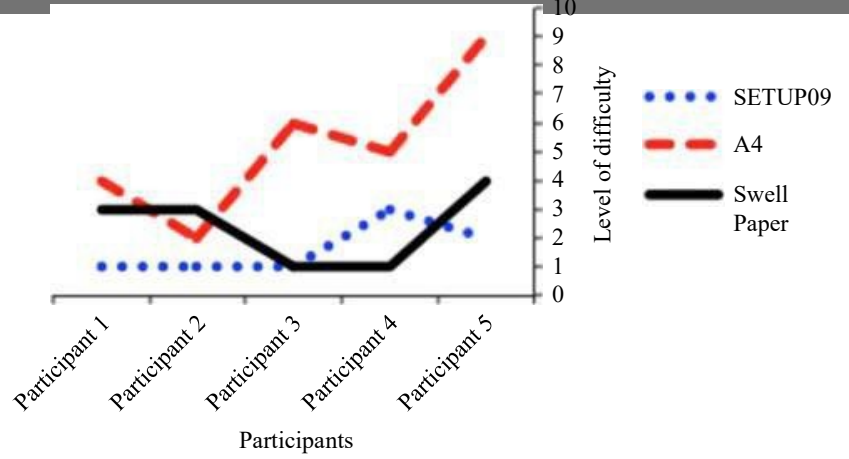


identified target locations of the tactile maps incorrectly when it went beyond three zoom levels. Attempts touse the virtual map for smaller points of identification and area sizes of the areawere difficult. They were able to tell the number of zoom levels by memory and found the path back to the root within three zoom levels. Observation demonstrates that they were competent with recalling the location to trace the routeback.

During theexecution of the firstsetofcommandswhenfindingthetargetlocations, theapplicants were successful in getting to the location with an error rate (28 percent) and with little time (33 s), without the help of a support worker. Participants commented that the "Compassbased navigation (SETUP09) system helped to understand the target location"; and also mentioned that it was important that "I am certain about my target location by touch or feel of the tactile map".

Another comment was that the questions such as "My position?" helped them to memorise the navigation better. Another participant commented, "The prototype needs practising several times before you really learn to use it". All participants agreed that they enjoyed the simple commands vocabulary to navigate using a mechanism that they are familiarwith and that theywould like it as a screen navigation technique.

Completion time and ease of use suggested that all participants were able to complete the commands in an average time of $33 \mathrm{~s}$. In total, 72 per cent of attempts were successful, and 28 per cent of attempts were unsuccessful. Observation demonstrates that compass-based navigation supports the blind participants to find target locations on the screen by building a navigation map. SETUP09 enables blind people to navigate to a particular location with confidence, without the support of a support worker. SETUP09 navigation system and raised line tactile maps both recorded the average time of 0.05 (ss) and 79 percent similarity of the time spent when using SETUP09 and tactile maps. There is no significant difference of the completion time between finding a location using the SETUP09 navigation system as opposed to using raised line tactile maps. However, SETUP09 is recorded to be more efficient than swell papers because it has taken less completion time, less cognitive load and better accuracy based on the participants' feedback than using a swell paper. The feedback is captured in the Table II.

Also there is a significant difference ( $p$ O 0.05 ) between correctly identifying a location using SETUP09 and incorrectly identifying a location using SETUP09 that demonstrates 72 per cent of the time there was a correct build of navigation maps in participants' minds. The main reason for the difference of data (incorrect recognition of target locations-activity 4, 5) is due to the tactile map complexity of higher zoom levels that will not impact when using SETUP09. Another reason for the difference of data is due to the unfamiliarity of the SETUP09 system, which can eventually be rectified.

\subsection{Limitations}

Several limitations were identified in the research. A larger cohort of BVI participants and greater prior familiarisation with the SETUP09 interface would have increased the power of the study. Touch input, voice input and hotkeys would have impacted on completion times of the exercises. More specific $\mathrm{HCl}$ usability questions such as IBM usability questions could have strengthened user feedback of the system.

Some participants commented on the ability to trustthe computerwithoutfeeling theneed to confirm the commands. The system currently does not show any magnification of the selected locations. This is potentially a feature worth adding for future experiments particularly for partially sighted users.

Table II Participants' comments: 1 - strongly agrees to 5 - disagree

SETUP09 compass-based navigation helped me to understand the target location better than swell papers The prototype needs practising several times before I use it with confidence I think my cognitive map is correctly built when using SETUP09 system

\begin{tabular}{l|l|l|l|l|}
1 & 1 & 1 & 2 & 1 \\
\hline 1 & 3 & 1 & 2 & 2 \\
\hline 1 & 1 & 1 & 1 & 1 \\
\hline
\end{tabular}


Some participants neededmore timegettingused to the system and commands. Therefore, different user levels could be an option at the startup screen. Many of the participants commented on the difficulty of swell paper grids recognition, especially beyond the second zoom level. They found it easier when the swell paper image was larger and less information was presented with variation and sufficient spacing. Therefore, the haptic (2D) images should be carefully planned before the print of the constructed images on the SETUP09 system.

In summary, they were much happier to use the SETUP09 system than the other presented methods. They all agreed to have confidently built the navigation map in their mind correctly according to the above results. A further possibility is to enlarge the zoomin command to favour partially sighted individuals. One participant did not like the time recording and the default narrator voice of the system. Compass-based navigation was easier to use when tracing the route back to the origin, as there were commands such as "My position?" However, conceptualising the size of the target area was not easy when it navigated beyond two zoom levels.

The questions and the comments of participants demonstrate the challenges of the SETUP09 system when the target location is small/or in multiple zoom levels, therefore, demonstrates difficulty tracing the size of the area. Even though compass-based navigation is proven to build a navigation map in BVI participants' minds, the experiments highlight that there is a need to develop more haptic feedback such as touch pads with vibrational feel of target location and sonification to improve the knowledge of a location and the size.

\section{Conclusion}

In this paper, compass-based screen navigation is discussed to help with computeraided drawing for BVI individuals. The navigation system is operated by compiler input commands. A compass on the interface takes the user into the intended location where the screen is divided into $3 \times 3$ memorable locations. The granularity is changed by zoomin and zoomout commands. BVI participants were tested with different screen navigation tasks to find accuracy and efficiency of compass-based navigation techniques by using a developed prototype (SETUP09) and tactile paper grid map. The task completion time was used to determine the efficiency of the system and error rate was used to determine the accuracy. User satisfaction and cognition have been taken into consideration in assessing the outcome of the experiment results. Our basic observation of compass-based navigation systems for blind computer users is that it is a good platform, proven to take minimum effort, time and resources to develop screen navigation.

Comparing the result between SETUP09 prototype and tactile paper grid maps, it can be observed that BVI participants shows high levels of accuracy and efficiency using SETUP09 to reach a target location, while less accuracy and efficiency were observed with use of the tactile grid map. It was difficult to find smaller locations on the tactile paper even when presented with a raised line grid system. Our observation shows that participants were faster and more accurate when finding smaller target areas with SETUP09 than tactile grids. Although hypotheses will need to be tested with a larger cohort, the initial findings are that the SETUP09 system enables BVI users to move from one location to another location with confidence, and without the need for the intervention of a support worker. Furthermore, it reveals that there is no significant difference between finding a location using the SETUP09 navigation system as opposed to using raised line tactile maps, proving that the SETUP09 navigation method is an efficient method to find a specific location on the screen. It was observed that there is a high level of accuracy reaching a specific location on the interface using the SETUP09 system compared to not reaching a given location on the interface. This indicates high cognition of the navigation concept among participants.

The compass-based grid over-layer could also be used with many more general and practical applications: not just as a navigation technique for drawing, but also as an easy navigation technique on the screen with everyday applications among sighted computer users. For example, to move the cursor to a specific location on a word processing application without having to use a mouse or tracker pad, but instead a compass grid over-layer with keyboard keys or speech. Another examplewould be the ability to move cursor to a location on a webpage for software such as screen readers. The reading can initiate or resume from the newly selected grid mouse location. A third example is to find a location on a map using $3 \times 3$ compassbased grid over layer. 
The technique traces the navigation path and remembers to navigate back and forth. Compass-based navigation takes the cursor into intended screen location without having to rely on a tracker pad, mouse or visual perception.

Future work will focus on extending easy command input mechanisms using keyboards, braille and speech. Even though speech is proven to be less accurate and time consuming (Sears et al., 2002), we hope that non-computer and braille users could benefit from it. SETUP09 is yet to be tested with different input interfaces and validation techniques. Additional information such as adding a sound layer and sound pitch to the interface to recognise the area of the screen and zoom level of the screen could work better if participants were familiar with musical vocabulary. The compass-based navigation can also be improved with many more lexica to trace the way back by extending compiler commands to accommodate more questions based on the orientation of the focus point or use of labels. Further investigation is required to explore BVI people's potentially limited perception and conception of three-dimensional space. However, haptic validation using mobile phones or hyper braille could be made available.

The next experiment will mainly focus on complex drawing mechanisms using the compiler commands of the SETUP09 system with compass-based navigation techniques to navigate the screen; for example, the ability of the SETUP09 system to draw a diagram or a flow chartoran image using compass-based navigation, as well as drawing commands with facilities such as user-defined shapes, primitive geometric shapes, grouping and manipulation of objects and labelling. In such cases efficiency, accuracy, ease of use and user satisfaction will need to be evaluated (Cook and Polgar, 2007).

\section{References}

Blenkhorn, P. and Evans, D.G. (1998), "Using speech and touch to enable blind people to access schematic diagrams”, Journal of Network and Computer Applications, Vol. 21 No. 1, pp. 17-29.

Calder, M., Cohen, R.F., Landry, J.L.N. andSkaff, J. (2007), “Teaching data structures to students who are blind”, 12th Annual SIGCSE Conference on Innovation and Technology in Computer Science Education', Vol. 39, ACM, SIGCSE, Dundee, 23-27 June.

Cook, A.M. and Polgar, J.M. (2007), Cook and Hussey's Assistive Technologies: Principles and Practice, Mosby, St Louis, Missouri.

de Mauro, C., Gori, M., Maggini, M. and Martinelli, E. (2001), "Easyaccess to graphical interfaces byvoice mouse”, technical report, Universit’a di Siena, SienaSI, available at: maggini@dii.unisi.it

Feng, J. and Sears, A. (2004), "Using confidence scores to improve hands-free speech based navigation in continuous dictation systems using confidence scores to improve hands- free speech based navigation in continuous dictation systems", $\underline{A C M}$ Transactions on Computer-Human Interaction, Vol. 11 No. 4, pp.329-56.

Hersh, M.A., Johnson, M.A. and Michael, A. (2008), Assistive Technology for Visually Impaired and Blind People, Number 1, Springer-Verlag, London and Berlin.

Kamel, H.M. and Landay, J.A. (2002), "Sketching images eyes-free: a grid-based dynamic drawing tool for the blind", Fifth International ACM Conference on Assistive Technologies, Number 33-40, ASSETS 02, ACM Press, Edinburgh.

Mcnair, A.E. and Waibel, A. (1994), "Improving recognizer acceptance through robust, natural speech repair", Proc. ICSLP, Vol. 3, pp. 1299-302.

Ohene-Djan, J. and Fernando, S. (2016), "Drawing for blind learners: assistive technology for graphical design", in IEEE(Ed.), IEEE16th International Conference onAdvanced Learning Technologies(ICALT), IEEE, Austin, Tx, 25-28 July, pp. 436-40.

Petrie, H., Schlieder, C., Blenkhorn, P., Evans, G.A., King, A.M.O., Ioannidis, G.T., Gallagher, B.D., Crombie, R.M. and Alafaci, M. (2002), "Tedub: a system for presenting and exploring technical drawings for blind people”, 8th International Conference ICCHP', Vol. 2398, ICCHP 2002, ACM Press, Linz.

Rita, B.Y. (2004), "Tactile sensory substitution studies", Annals of the New York Academy of Sciences, Vol. 1013 No. 1, pp. 83-91. 
Sears, A., Lin, M. and Karimullah, A. (2002), "Speech-based cursor control: understanding the effects of target size, cursor speed, and command selection", Universal Access in the Information Society, Vol. 2, pp. 30-43.

Sears, A., Karat, M., Oseitutu, K., Karimullah, A. and Feng, J. (2001), "Productivity, satisfaction, and interaction strategies of individuals with spinal cord injuries and traditional users interacting with speech recognition software", Universal Access in the Information Society, Vol. 1 No. 1, pp. 4-15.

Suhm, B., Myers, B. and Waibel, A. (2001), "Multimodalerrorcorrectionfor speech userinterfaces", $\underline{A C M}$ Transactions on Computer-Human Interaction, Vol. 8 No. 1, pp. 60-98.

Takagi, N. (2009), "Mathematical figure recognition for automationg production of tactile graphics", IEEE International Conference on System, Man and Cybernetics (SMC 2009), pp. 4651-6.

Williams, G.J., Zhang, T., Lo, A., Gonzales, A., Baluch, D.P. and B.S.D. (2014), "3D printing tactile graphics for the blind: application to histology", Annual Rehabilitation Engineering Society of North America Conference 2014, Indianapolis, IN.

Zhu, S. and Feng, J. (2010), "Investigating grid-based navigation: the impact of physical disability", ACM Transaction on Accessible Computing, Vol. 3 No. 1, pp. 3-30.

\section{About the authors}

Dr James Francis Ohene-Djan conducts research in the areas of assistive technologies, social video, adaptive web systems, personalised interaction and $\mathrm{HCl}$. Dr Ohene-Djan's research has resulted in 40 refereed publications in international journals and conference proceedings and has been presented at 30 international conferences. Dr Ohene-Djan has acted as session chair and review committee member for the IEEE International Conference series on Advanced Learning Technologies. Dr James Francis Ohene-Djan is the corresponding author and can be contacted at: j.djan@gold.ac.uk

Sandra A. Fernando's research is focussed on development of computer-aided drawing techniques and technologies for blind students. Having previously taught blind students and developed learning technologies for blind interaction, she now focusses on formally modelling the creative processes behind blind users drawing. Sandra Fernando's research is supported by a grant from MACS charity, UK (www.macs.org.uk/). 